\title{
Power generation by using piezoelectric transducer with bending mechanism support
}

Anis Maisarah Mohd Asry, Farahiyah Mustafa, Maizul Ishak, Aznizam Ahmad

Faculty of Engineering Technology, Universiti Tun Hussein Onn Malaysia, Malaysia

\begin{tabular}{l}
\hline Article Info \\
\hline Article history: \\
Received Sep 17, 2018 \\
Revised Nov 29, 2018 \\
Accepted Dec 17, 2018 \\
\hline
\end{tabular}

Keywords:

3D model

Bending mechanism

Deflectio

Piezoelectric transducer

Renewable energy

\begin{abstract}
This paper presents about power generation by using piezoelectric transducer with bending mechanism support. In this study, bending mechanism is developed by employing 3D printer technology. This 3D model is used as a support for a piezoelectric transducer during deflection or bending process. During deflection condition, stress that applied on the piezoelectric transducer will generate electrical energy. The 3D model helps the piezoelectric transducer to produce more voltage output. A finger press test used as evaluation method for the voltage output of the piezoelectric transducer. The experiment is tested by varying three different 3D model with the different diameter for the middle hole for each of the model. A round shape of the piezoelectric transducer with size of $50 \mathrm{~mm}$ in diameter is used to conduct the experiment. Thus, when the piezoelectric transducer placed on the 3D model with $0 \mathrm{~mm}$ in diameter of middle hole will producing $5.4 \mathrm{~V}$ voltage output. However, 3D model with $30 \mathrm{~mm}$ diameter of middle hole, the output increases up to $19.0 \mathrm{~V}$. The output voltage for piezoelectric transducer reached its highest voltage when placed on the 3D model with middle hole of $40 \mathrm{~mm}$ which is $34.4 \mathrm{~V}$. This bending mechanism can be used to increase the output of piezoelectric transducer as it applied underneath footstep tile at crowded area to harvest the energy produced from walking activities. The power generated can be used to power up various electronic devices.
\end{abstract}

Copyright (C) 2019 Institute of Advanced Engineering and Science. All rights reserved.

\section{Corresponding Author:}

Farahiyah Mustafa, Faculty of Engineering Technology, Universiti Tun Hussein Onn Malaysia, Hub Pendidikan Tinggi Pagoh, 86400 Pancur, Johor, Malaysia.

Email: farahiyah@uthm.edu.my

\section{INTRODUCTION}

In our daily activities, energy has became the most basic need due to energy demand has rising up day by day. This happened because of the growth of human population nowadays. Sources of energy these days are insufficient to support the demand. In order to solve this problem, another alternative sources need to be produced to support the insufficient sources. The best way is by implementing renewable energies. These energies need to be harvest before being used. Energy harvesting that also known as energy scavenging is the process of energy capturing or deriving the energy from external sources, accumulating and storing the energy for the later use [1], [2].

By harvesting this renewable energy, the electricity can be generated without giving negative impacts on the environment. The quality of human life is increased due to the development of medical technology and the growth of the human population. The energy that can be generated to fulfil the demand needs through the growth of human population. By using the human footstep, useful energy can be harvest and create electricity for non-renewable energy replacement. This type of renewable energy does not depending or relying on climate or weather condition. In order to invent a free pollution technology, a 
transducer is used to harvest the energy source. The transducer used is piezoelectric transducer. This transducer is used to detect vibration or pressure that applied on it to generate electricity [3]. This piezoelectric converts mechanical energy exerted by pressure or force into electrical energy and used for other small electronic devices or being stored in the storage compartments [4].The output voltage and current that generated from piezoelectric transducer are small.

The piezoelectric transducer is completely depending on crystalline structure. This piezoelectric transducer consists of positive and negative charges. Therefore, when the force or pressure are applied, magnitude of the polarization can be determined. During normal condition or without stress or strain condition, piezoelectric transducer will stay with neutral charge as there is no potential difference produced when negative and positive charges is balanced for both sides [5].

Direct piezoelectric effect and converse piezoelectric effect are two main category of piezoelectric effect [5], [6]. The direct piezoelectric effect is also known as generator or transducer effect to illustrate the ability by converting mechanical energy into electrical energy [5]-[7]. On the other hand, the converse piezoelectric effect is the inverse ability of the direct piezoelectric effect which it converts electrical energy into mechanical energy [5], [7], [8]. This converse piezoelectric effect also known as motor or actuator effect.

The piezoelectric transducer converts the mechanical energy from the pressure that applied on it to generate the electrical energy by employing the piezoelectric effect has received a considerable research attention. Currently, the studies focus on using a single piezoelectric module. There are some researches have been conducted by developing a prototype that utilizing the human footstep that strikes to the piezoelectric bean which developed by M. Renaud and his team [9]. Their research results showed $600 \mu \mathrm{W}$ harvested from the $10 \mathrm{~Hz}$ frequency and $10 \mathrm{~cm}$ amplitude linear motion. Another research conducted by M. Ferrari et.al has been improved by coming out with a prototype of an autonomous battery-less sensor module [10]. This module successfully harvested $35 \mathrm{~mW}$ of electrical power and power up the sensor module and send the data within a range of $25 \mathrm{~m}$ in a laboratory environment.

A.M. Abdal and his team created the piezoelectric pre-stressed bending mechanism for ImpactDriven Energy Harvester [11]. This pre-stressed piezoelectric bending mechanism is designed to further improve and maximize the output wattage generated by piezoelectric comparing to unstressed piezoelectric. This piezoelectric can harvest around $70 \mathrm{AC} \mathrm{V}$ at open circuit measurement by using suggested mechanism and after rectifying the maximum DC power of $53 \mathrm{~mW}$ with a load of $700 \Omega$. Kyoon N.C and Hee H. R. presented a novel impact based piezoelectric harvester mechanism. In this design, the continuous impact on the piezoelectric surface is applied by using wind flow and it successfully harvests a continuous electricity by implement this method [12]. The improvement that the author tried to do is increasing the impact by using a spring retention action to increase the frequency in order to improve its efficiency. P. Madhu and her team designed a footstep tile by using piezoelectric transducer [13]. In this research, they are using 72 piezoelectric transducers and 72 plastic bushes that are attached to the bottom surface of the top plate. This model produces an output voltage range of $10-15 \mathrm{~V}$ and outputs current range of $50-400 \mu \mathrm{A}$.

This paper presents power generation by using piezoelectric transducer with bending mechanism support. In this study, the piezoelectric transducer is used. The output voltage from the piezoelectric transducer is low. In order to maximize the output voltage, the bending mechanism has been designed. A 3D model with a middle hole is designed to give space and support to the piezoelectric transducer during the bending process. This 3D model provides space for the piezoelectric transducer to increase its deflection as it also increases the electrical energy that had been generated. The $3 \mathrm{D}$ model is designed to place this transducer within it and the middle hole of 3D model is designed with the different diameter to find the most perfect 3D model to help piezoelectric transducer increasing its deflection and output voltage.

\section{RESEARCH METHOD}

In this study, lead zirconate titanate (PZT) piezoelectric transducer is used to harvest the mechanical energy produced when force or pressure are applied onto it and converts into electrical energy. The output of this transducer depends on the magnitude of stress and strain that applied on its structure. The round shape piezoelectric transducers are used in this experiment. The piezoelectric transducer has the diameter of $50 \mathrm{~mm}$ crystalline structure. This piezoelectric transducer common output voltage can achieves around $0-12 \mathrm{~V}$ at normal generate. However, during instant impact, it can reaches up to $30 \mathrm{~V}$ and $5 \mathrm{~mA}$. The vibration manner that is more suitable for round shape piezoelectric transducer is by pressing, pulse and shock. The piezoelectric transducer produces low electrical energy and its output depending on the pressure, force or stress that being hit on it. This piezoelectric transducer will be deflected when there are pressure or stress applies to it.

The output of this piezoelectric transducer is very low. In order to enhance the output from the piezoelectric transducer, it needs some support to maximize the deflection process. This support is the

Power generation by using piezoelectric transducer with bending mechanism ... (Anis Maisarah Mohd Asry) 
bending mechanism that designed by using solid work software and print in 3 dimensional (3D) size. It is used as a support during the bending process of the piezoelectric transducer when force or pressure are applied to it. This 3D model design is based on the shape and size of the piezoelectric transducer that is used. The three different type of 3D models are designed to test the bending process of piezoelectric transducer. The difference between these three models is the diameter measurement of its middle hole. The diameter of the middle hole for the first 3D model is $0 \mathrm{~mm}$ or 3D model without the middle hole. The second 3D model has $30 \mathrm{~mm}$ in diameter of the middle hole and the third 3D model is $40 \mathrm{~mm}$ in diameter of the middle hole. The middle hole for the 3D model provides space for the bending process of the piezoelectric transducer.

The experiment is conducted to test the performance of $3 \mathrm{D}$ model during the bending process. The testing process of the piezoelectric transducer is tested by using finger press. The piezoelectric transducer is placed in a 3D model and being pressed to produce electric energy The subject with 60kg body weight is assigned for the finger press activities on the piezoelectric transducer. The finger is press on top of the piezoelectric transducer to determine the voltage output of a single cell of the piezoelectric transducer. The three 3D models with different measurement of its middle hole diameter which are $0 \mathrm{~mm}, 30 \mathrm{~mm}$ and $40 \mathrm{~mm}$ are tested in this study. Figure 1 shows the three different types of 3D model.

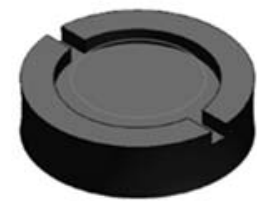

(a)

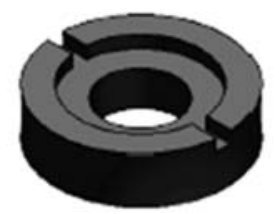

(b)

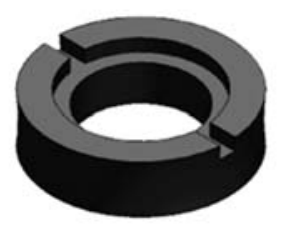

(c)

Figure 1. The 3D model with different diameter (a) $0 \mathrm{~mm}$, (b) $30 \mathrm{~mm}$, (c) $40 \mathrm{~mm}$

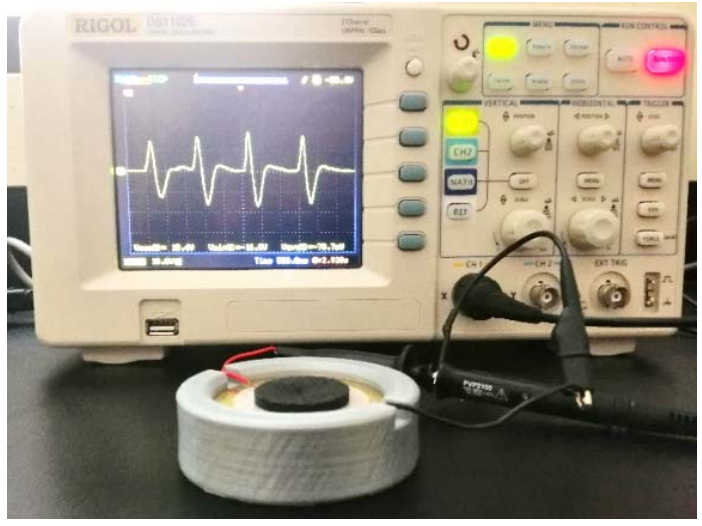

Figure 2. The set up for testing the bending process piezoelectric transducer

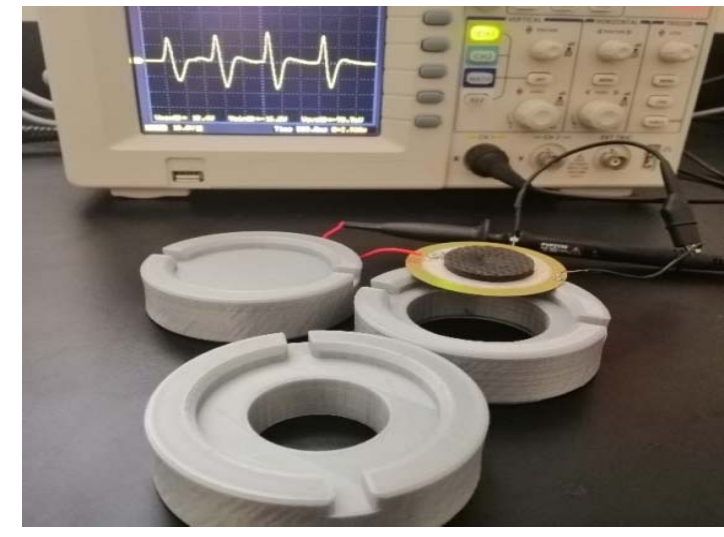

Figure 3. The set up for testing with three 3D model with the different diameter

\section{RESULTS AND ANALYSIS}

The piezoelectric transducer output is an AC waveform. Each piezoelectric is placed on the 3D models with different middle hole diameter and it produced a sinusoidal transient pulse. The oscilloscope that is connected to the piezoelectric transducer is to measure the voltage output. The output from the piezoelectric transducer that placed on the 3D model is different for each $3 \mathrm{D}$ model. This is because the bending process of the piezoelectric transducer depends on the size of the middle hole in the 3D model. This middle hole in the 3D model provides space for the piezoelectric transducer when the bending process took place. Figure 4 shows the output voltage when the finger press test is applied on the piezoelectric transducer that placed on the $3 \mathrm{D}$ model with $0 \mathrm{~mm}$ in diameter. 


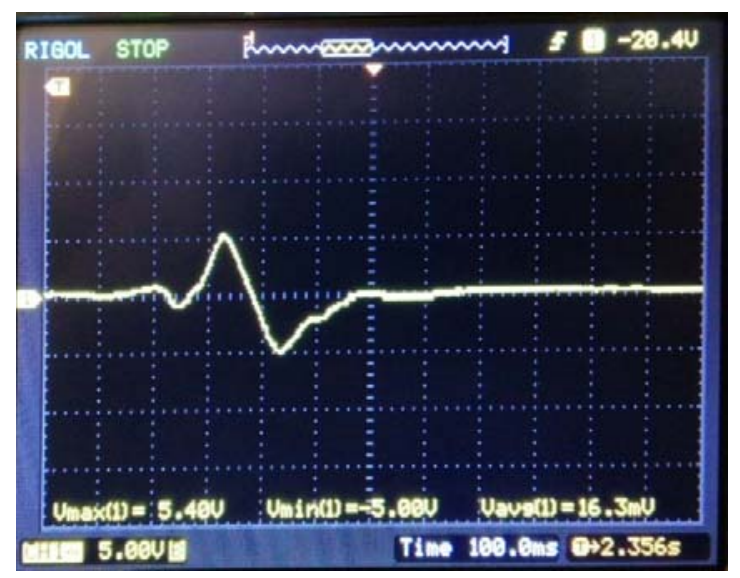

Figure 4. The voltage output waveform for the piezoelectric transducer when placed in a 3D model with $0 \mathrm{~mm}$ in diameter

The output voltage for the piezoelectric transducer with the 3D model without the middle hole or 0 $\mathrm{mm}$ in diameter only produces $5.40 \mathrm{~V}$ in $\mathrm{AC}$ form. In this $3 \mathrm{D}$ model, the piezoelectric transducer cannot bend too much as there is no space for the transducer to deflect when there are force or pressure applied on it. The voltage that is generated is also low depending on how much the deflection can take place. The piezoelectric transducer produces an $\mathrm{AC}$ waveform. The output of the piezoelectric transducer will be rectified before being stored or used to power up low power devices. The scope of this testing is only to enhance the voltage output of the piezoelectric transducer before it will further used for footstep application. Figure 5 shows the output voltage of the piezoelectric transducer produced by the finger compression force when it is placed on the 3D model with $30 \mathrm{~mm}$ in diameter.

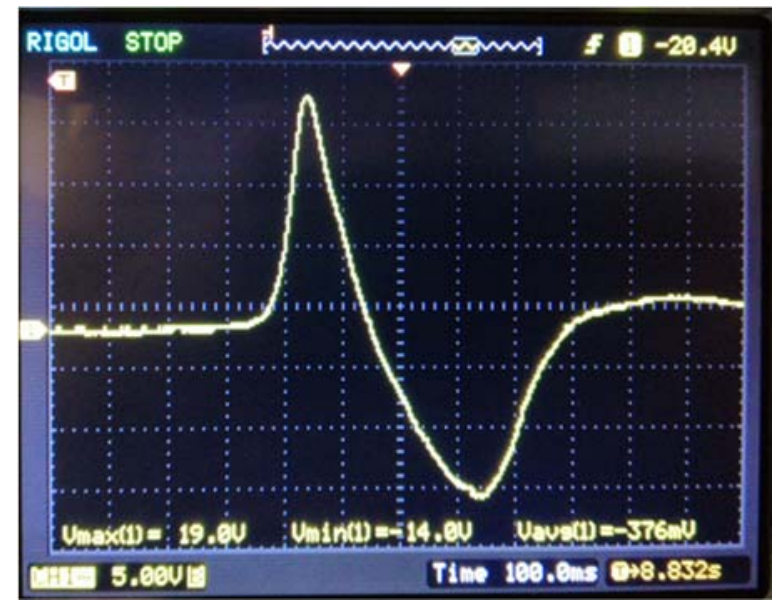

Figure 5. The voltage output waveform for the piezoelectric transducer when placed in a 3D model with $30 \mathrm{~mm}$ in diameter

The output voltage in Figure 5 shows that the voltage is $19.0 \mathrm{~V}$. The output voltage for this $3 \mathrm{D}$ model is higher compared to the 3D model without the middle hole. The middle hole in this 3D model provides space for the piezoelectric transducer to bend and support it when there is pressure applied on it. The bending process causes the piezoelectric transducer to generate electricity by converting the mechanical energy into electrical energy. The middle hole with $30 \mathrm{~mm}$ in diameter makes the bending process much easier compared to the 3D model without the middle hole. Figure 6 shows that the output voltage of the piezoelectric transducer produced when placed on the 3D model with $40 \mathrm{~mm}$ in diameter.

Power generation by using piezoelectric transducer with bending mechanism ... (Anis Maisarah Mohd Asry) 


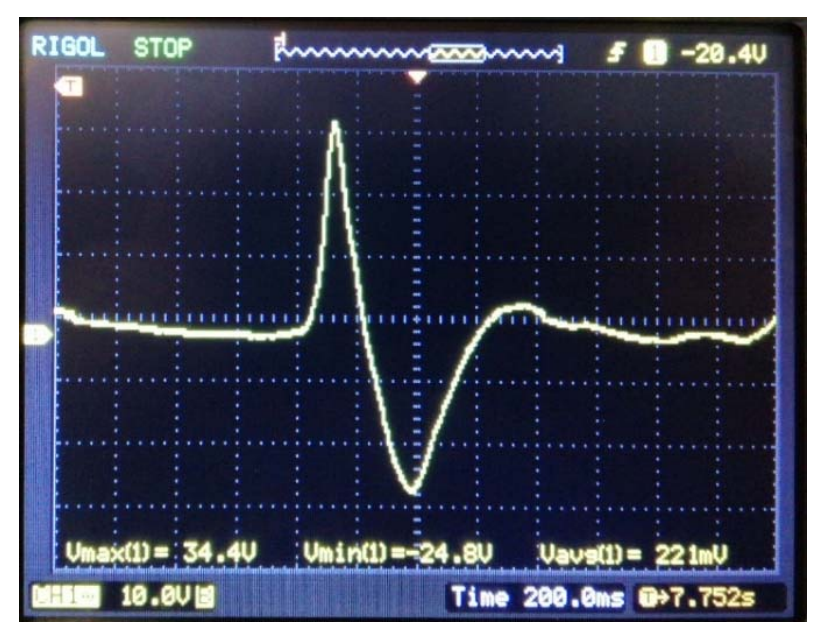

Figure 6. The voltage output waveform for the piezoelectric transducer when placed in a 3D model with $40 \mathrm{~mm}$ in diameter

For this $3 \mathrm{D}$ model, the output voltage that generated from the piezoelectric transducer is $34.4 \mathrm{~V}$. It shows that the output voltage generated when the piezoelectric transducer is placed in the 3D model with 40 $\mathrm{mm}$ in diameter is the highest output voltage compared to other two 3D models. The space for this 3D model is bigger compared to the other two 3D models. The bending process of piezoelectric transducer that takes place in this 3D model has more space and support when it deflects or bends during the finger press test. This space and support allow the piezoelectric transducer to generate more output voltage compared to the output voltage for the other two of the 3D models. As conclusion, the space between the $3 \mathrm{D}$ models plays an important role in generating more output voltage of the piezoelectric transducer. The diameter of the middle hole for the 3D model should match and fit with the size of the piezoelectric transducer so that this 3D model could provides the suitable space and support for the piezoelectric transducer when the bending process happened.

\section{CONCLUSION}

This paper has presented power generation by using piezoelectric transducer with bending mechanism support. In this study, a research on how to generate more voltage output from the piezoelectric transducer is conducted because the output voltage of the piezoelectric transducer is very low. The $3 \mathrm{D}$ model is designed as the piezoelectric transducer need to undergo bending process in order to generate more voltage. Piezoelectric transducer is capable of producing voltage output by using the $3 \mathrm{D}$ model as a support. The 3D model with an accurate diameter for the middle hole allows the piezoelectric transducer to bend more and it generates a high voltage output. The 3D model with $40 \mathrm{~mm}$ in diameter for the middle hole exerts 34.4 $\mathrm{V}$ which is the highest voltage output rather than other two 3D models. Hence, this result can be used to form a piezoelectric tile to generate more power using the human footstep. In this research, the testing that is conducted to enhance the voltage output of the piezoelectric transducer before it will further used for footstep application. This tile in further can be placed at crowded area as the piezoelectric transducer is very sensitive to force and pressure. The walking energy can be harvest and becomes a useful energy when the piezoelectric tile is placed at that area. It is specifically suitable to be implemented at pavement street, train ticket counter, stairs and dance floor. The piezoelectric tile is also suitable for the exercise tile such as for skipping or on the treadmill. The power that is generated from this piezoelectric tile can be used to power up the light street, light along the stairs and also low power appliances.

\section{ACKNOWLEDGEMENTS}

The authors gratefully acknowledge the support of the Malaysian Ministry of Higher Education (MOHE) through FRGS Research Grant No. 1615 and the Universiti Tun Hussein Onn Malaysia through Short - Term Grant No. U541.

Int J Pow Elec \& Dri Syst, Vol. 10, No. 1, March 2019: $562-567$ 


\section{REFERENCES}

[1] E. Maghsoudi, et al., "A review of walking energy harvesting using piezoelectric materials," International Conference on Architecture and Civil Engineering (ICACE 2017), pp. 1-8, 2017.

[2] A. Adhithan, et.al., "Proposed Method of Foot Step Power Generation using Piezo Electric Sensor," International Advanced Research Journal in Science, Engineering and Technology, vol. 2, pp. 25-29, April 2015.

[3] M.N.Gupta, et al., "Electricity Generation Due to Vibration of Moving Vehicles Using Piezoelectric Effect," Electricity Generation Due to Vibration of Moving Vehicles Using Piezoelectric Effect, vol. 4 pp. 313-318. 2014.

[4] J. Varghese and P. Karikottil, "Footstep Power Generation using Piezoelectric Sensors," International Journal of Recent Innovation in Engineering and Research, vol. 2, pp. 11-18. August 2017.

[5] D. Vatansever, et al., Alternative Resources for Renewable Energy: Piezoelectric and Photovoltaic Smart Structures, Global Warming - Impacts and Future Perspectives, 2012, pp. 264-268.

[6] P. Arora et al., "Piezoelectrics - A Potential Electric Source for Aircrafts," Proceedings of the World Congress on Engineering, pp. 978-980, 2013.

[7] V. Prasannabalaji, et al., "Staircase Power Generation Using Piezo-Electric Transducers," Advance in Electronic and Electric Engineering, vol. 3, pp. 747-754, 2013.

[8] A. Kokkinopoulos, et al., "Energy harvesting implementing embedded piezoelectric generators - The potential for the Attiki Odos traffic grid," TerraGreen 13 International Conference 2013 - Advancements in Renewable Energy and Clean Environment, pp. 1-17, 2013.

[9] M. Renaud, P. Fiorini, R. van Schaijk and C. van Hoof, "An impact based piezoelectric harvester adapted to low frequency environmental vibrations," TRANSDUCERS 2009 - 2009 International Solid-State Sensors, Actuators and Microsystems Conference, pp. 2094-2097, 2009.

[10] M. Ferrari, et al., "Portable energy-logger circuit for the experimental evaluation of energy harvesting solutions from motion for wearable autonomous sensors," Eurosensors 2014 Procedia Engineering 87, 2014 , pp1230 -1233.

[11] A. M. Abdal and K.S. Leong, "Piezoelectric Pre-Stressed Bending Mechanism for Impact Driven Energy Harvester," International Technical Postgraduate Conference IOP Conf. Series: Materials Science and Engineering 210, pp 1-11, 2017.

[12] N. Kyoo and H.R. Hee, "Continuous Energy Harvesting Method Using Piezoelectric Element", 2015 IEEE 2nd International Future Energy Electronics Conference (IFEEC), pp 1-4, 2015.

[13] P. Madhu, et al., "Electrical Power Generation by Foot-steps using Piezo-electric Transducers," International Journal of Recent Trends in Engineering \& Research (IJRTER) vol. 2 pp 108 - 115. 